\title{
Appropriate and Inappropriate Implantable Cardioverter Defibrillators Therapies in Arrhythmogenic Right Ventricular Cardiomyopathy/Dysplasia Patients
}

\author{
Bandar Al-Ghamdia, b, c , Yaseen Mallawi a, b, Azam Shafquat ${ }^{\mathrm{a}, \mathrm{b}}$, Nadiah AlRuwailia, \\ Ayman Alhazaymeha, Waleed Al-Manea ${ }^{\mathrm{a}}$, \\ Majid Al-Fayyadh ${ }^{\mathrm{a}}$
}

\begin{abstract}
Background: Arrhythmogenic right ventricular cardiomyopathy/ dysplasia (ARVC/D) is an inherited cardiomyopathy characterized histologically by the replacement of ventricular myocardium with fibrous and fatty tissue, and clinically by ventricular tachycardia arrhythmias primarily of right ventricular (RV) origin. Implantable cardioverter defibrillator (ICD) is the only proven therapy to reduce mortality in ARVC/D patients. However, it has the risk of inappropriate anti-tachycardia pacing (ATP) or shocks. This study aimed to assess the occurrence of appropriate and inappropriate ICD therapies in ARVC/D patients who underwent ICD implantation in a single Cardiac Centre.
\end{abstract}

Methods: Retrospective analysis of the data of patients with the diagnosis of ARVC/D based on the 2010 revised Task Force Criteria, who underwent ICD implantation in the Heart Centre, at King Faisal Specialist Hospital and Research Center (KFSH\&RC), Riyadh between January 1997 and May 2016. The clinical data and information about appropriate and inappropriate ICD therapies were obtained from medical records with the review of the available intra-cardiac electrograms (EGMs).

Results: Twenty-two ARVC/D patients with ICD implantation (20 males (91\%), mean age at ICD implantation: $32 \pm 14$ years). ICD was implanted for secondary prevention of sudden cardiac death (SCD) in 15 patients $(68.2 \%)$, and for primary prevention in 7 patients $(31.8 \%)$. At mean follow-up of $9.4 \pm 4.8$ years, 11 patients $(50 \%)$ had appropriate ICD therapies, and five patients $(22.7 \%)$ had inappropriate ICD therapies. Out of 950 ICD therapies, $865(91 \%)$ were appropriate (586 episodes of VT/VF treated with ATP $(61.3 \%)$, and 279 episodes treated with shocks $(29.37 \%))$ and $85(9.4 \%)$ were

Manuscript submitted May 28, 2018, accepted June 21, 2018

${ }^{a}$ Heart Centre, King Faisal Specialist Hospital and Research Centre (KFSH\&RC), Riyadh, Saudi Arabia

${ }^{\mathrm{b}}$ Alfaisal University, Riyadh, Saudi Arabia

${ }^{\mathrm{c} C}$ Corresponding Author: Bandar Al-Ghamdi, Heart Centre, King Faisal Specialist Hospital and Research Centre, MBC-16, PO Box 3354, Riyadh 11211, Saudi Arabia. Email: balghamdi@kfshrc.edu.sa

doi: https://doi.org/10.14740/cr734w inappropriate (45 episodes treated with ATP (4.73\%), and 40 treated with shocks $(4.21 \%))$.

Conclusion: ARVC/D patients are at risk of VT/VF arrhythmias. ICD therapy is the only proven life-saving therapy in those patients. Most of ICD therapies in our patient's population are appropriate, and ATP therapy is effective in terminating most of VT episodes. Although we do not have any patient with subcutaneous ICD, the high success rate of ATP suggests that transvenous ICD would be more appropriate in ARVC/D patients.

Keywords: Implantable cardioverter defibrillator; Arrhythmogenic right ventricular cardiomyopathy; Shocks; Anti-tachycardia pacing

\section{Introduction}

Arrhythmogenic right ventricular cardiomyopathy/dysplasia (ARVC/D) is an inherited cardiomyopathy characterized histologically by the replacement of ventricular myocardium with fibrous and fatty tissue, and clinically by ventricular arrhythmias primarily of right ventricular $(\mathrm{RV})$ origin $[1,2]$. The diagnosis of ARVC/D is based on a combination of diagnostic criteria proposed by the International Task Force for Cardiomyopathy which has been published initially in 1994 [3] then revised in 2010 [4]. Patients with ARVC/D usually present with symptoms due to ventricular arrhythmias, such as palpitations, dizziness, chest discomfort or syncope $[1,2]$. ARVC/D patients are at risk of sudden cardiac death (SCD) caused by sustained ventricular tachycardia (VT) or ventricular fibrillation (VF) which may be the initial presentation of ARVC/D [5]. Implantable cardioverter defibrillator (ICD) is the only proven therapy to reduce mortality in ARVC/D patients. However, it is not without risks that include inappropriate interventions with anti-tachycardia pacing (ATP) or shocks $[6,7]$.

The objective of this study is to assess the occurrence of appropriate and inappropriate ICD therapies in ARVC/D patients who underwent ICD implantation in the Heart Center, King Faisal Specialist Hospital and Research Center (KFSH\&RC), Riyadh. 
Table 1. Clinical Characteristics of the Patients

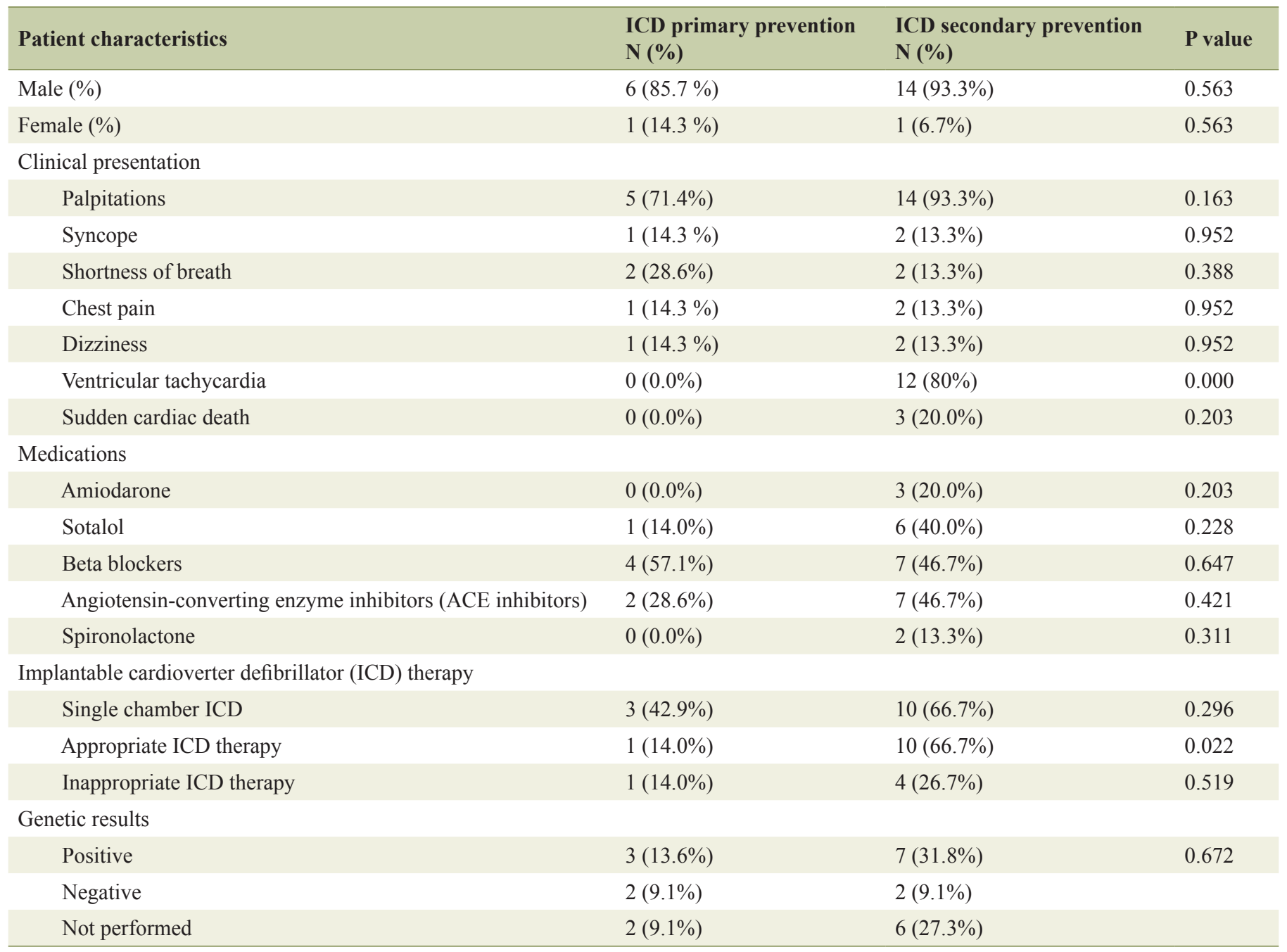

\section{Materials and Methods}

\section{Patients' selection}

Twenty-two ARVC/D patients were diagnosed based on 2010 task force criteria who underwent ICD implantations in our Heart Center between January 1997 and May 2017. The patients had regular follow-up in arrhythmia and device clinic every 6 months. The clinical data were collected from the patients' medical records and the device clinic data. This study was approved by the institutional review board of the KFSH\&RC.

\section{ICDs and classification of discharges}

All patients received multifunctional third- or fourth-generation ICDs. The managing cardiologist/electrophysiologist made decisions regarding ICD implantation and programming. Stored intracardiac electrograms were analyzed to classify arrhythmias responsible for precipitating defibrillator discharges, according to following definitions [8]: VF as tachycardia with a mean cycle length (CL) of $\leq 240 \mathrm{~ms}$ (irregular or regular); VT as a regular tachycardia with mean CL $>240 \mathrm{~ms}$. Defibrillator therapies were considered appropriate versus inappropriate based on standard criteria [5]. VT storm was defined as the occurrence of VT or VF that resulted in $\geq 3$ ICD interventions (shock or ATP) in a $24-\mathrm{h}$ period [9]. ICDs interrogations were performed routinely 6 - 8 weeks after initial ICD implantation and then every 6 months as per device clinic routine follow-up schedule.

\section{Definitions}

Syncope was defined as a transient loss of consciousness and postural tone with spontaneous recovery. Non-sustained ventricular tachycardia (NSVT) was defined as $\geq 3$ consecutive ventricular premature beats with a rate $>100$ beats/min, lasting $<30 \mathrm{~s}$ and without hemodynamic instability. NSVT was documented during exercise testing, loop monitoring or 24-h Holter monitoring. 


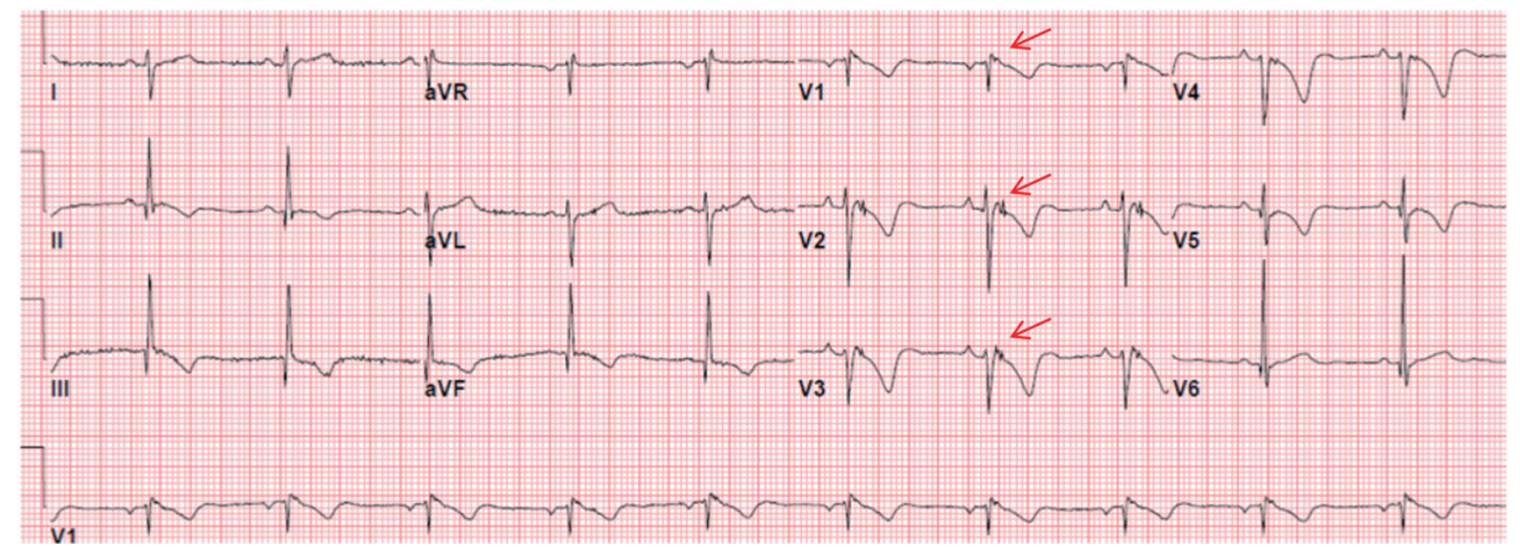

Figure 1. ECG of a patient with ARVC/D showing the presence of an epsilon wave (electric potentials after the end of the QRS complex) (red arrows) and T-wave inversion in V1 - V5.

SCD was defined as natural death from cardiac causes, heralded by abrupt loss of consciousness within $1 \mathrm{~h}$ of the onset of an acute change in cardiovascular status with or without pre-existing heart disease $[10,11]$.

Primary prevention of SCD referred to use of ICDs in individuals who are at risk for but have not yet had an episode of sustained VT, VF or cardiac arrest. Secondary prevention referred to an indication for an ICD exclusively for patients who have survived one or more cardiac arrests or sustained ventricular tachycardia [12].

\section{Statistical analysis}

Continuous data are expressed as mean $\pm \mathrm{SD}$ and compared across groups using a Student's $t$-test. Categorical variables are reported as frequency (\%) and compared between groups by the chi-square or Fisher's exact test. IBM SPSS Statistics version 20 was used for the analyses.

\section{Results}

The clinical characteristics of the patients are summarized in Table 1. The patients' age ranged from 13 to 67 years with a mean age of $41.9 \pm 17$ years, and 20 patients were males (90.9\%). The age at ICD implantation ranged from 12 to 54 years with a mean of $32 \pm 14$ years. The presenting symptoms were palpitations in $19(86.4 \%)$, syncope in $3(13.6 \%)$, shortness of breath in $4(18 \%)$, chest pain in $3(13.6 \%)$ and dizziness in 3 patients $(13.6 \%)$. Thirteen patients had documented VT on initial presentation (59\%), and three were survivors of SCD (13.6\%).

One patient died 10.5 years after ICD implantation due to advanced heart failure and other comorbidities.

\section{Electrocardiogram (ECG) abnormalities}

ECG showed depolarization abnormalities (epsilon wave-electric potentials after the end of the QRS complex) in 6 patients
(27.3\%), repolarization abnormalities with T-wave inversion in V1-3 or beyond in 15 patients $(68 \%)$ and $\mathrm{T}$-wave inversion in V1-2 or V4, 5 or 6 in 2 patients (9\%) (Fig. 1). Signal-averaged ECG was performed in three patients (13.6\%), and two had at least one minor criterion for ARVC/D diagnosis (filtered QRS duration (fQRS) $\geq 114 \mathrm{~ms}$, duration of terminal $\mathrm{QRS}<$ $40 \mu \mathrm{V}$ (low-amplitude signal duration) $\geq 38 \mathrm{~ms}$ or root-meansquare voltage of terminal $40 \mathrm{~ms} \leq 20 \mu \mathrm{V}$ ). Table 2 shows a summary of ECG changes [4] and Figure 2 shows clinical VT documentation in one of our patients.

Holter monitoring was performed in 11 patients $(50 \%)$, and it showed $>500$ ventricular extrasystoles per $24 \mathrm{~h}$ in six patients $(54.5 \%)$, and non-sustained VT in four patients (36.4\%).

\section{Cardiac imaging abnormalities}

By 2D echocardiogram; 10 patients (45.5\%) fulfill major criteria, and 4 patients (18\%) fulfill minor criteria for the ARVC/D diagnosis based on 2010 task force criteria [4]. RV function was normal in nine patients $(41 \%)$, mildly reduced in four patients $(18.2 \%)$, mildly to moderately reduced or moderately reduced in two patients (9\%), and moderately to severely reduced or severely reduced in seven patients $(31.8 \%)$. Left ventricular ejection fraction (LVEF) was normal (EF $>50 \%$ ) in 16 patients (72.7\%), mildly reduced (EF: $40-49 \%)$ in 4 patients $(18.2 \%)$, moderately reduced (EF: $30-39 \%)$ in one patient (4.5\%), and severely reduced $(\mathrm{EF}<30 \%)$ in one patient $(4.5 \%)$ (Fig. 3 [13]). The echocardiogram changes are summarized in Table 3 [4].

Cardiac magnetic resonance imaging (MRI) was performed in 12 patients $(54.5 \%)$; major diagnostic criteria were present in four patients $(33.3 \%)$, and minor diagnostic criteria were present in two patients (16.7\%) (Fig. 4 [13]). The cardiac MRI changes are summarized in Table 4 [4].

\section{ICD therapy}

The ICD was implanted for secondary prevention of SCD in 15 patients $(68.2 \%)$, and for primary prevention in 7 patients 
Table 2. Summary of ECG Changes

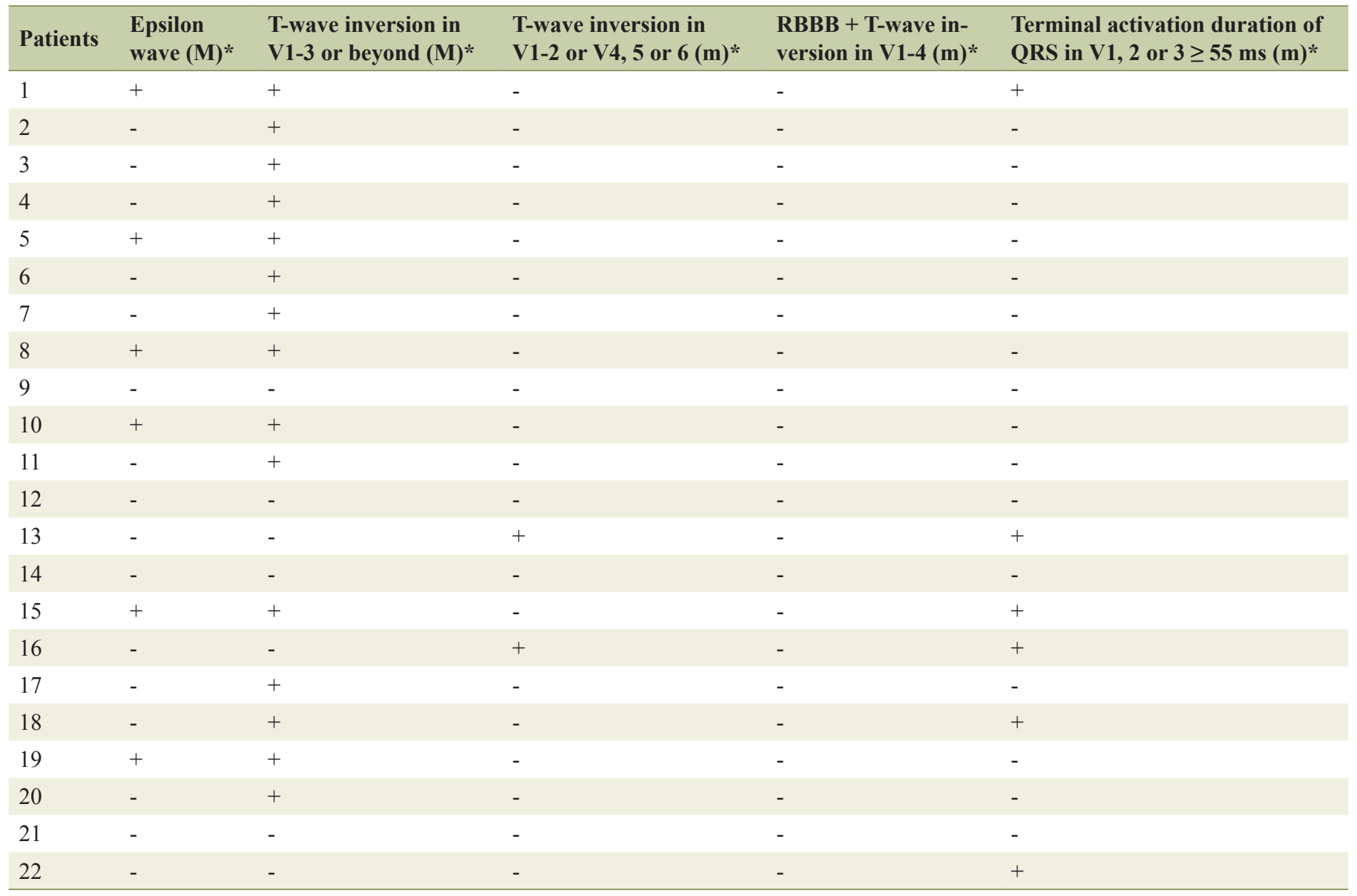

* $\mathrm{M}$ indicates major, and $\mathrm{m}$ indicates minor diagnostic criterion based on 2010 ARVC/D Task Force Criteria [4]. RBBB: right bundle-branch block. -: absent; +: present.

(31.8\%). Thirteen patients had a single chamber (59\%), and nine patients had a dual chamber ICD (41\%).

Over the follow-up period of $9.4 \pm 4.8$ years $(2-20.9$ years); 11 patients $(50 \%)$ had appropriate ICD therapies, and five patients $(22.7 \%)$ had inappropriate ICD therapies. Out of 950 ICD therapies, 865 (91\%) were appropriate (586 episodes of VT/VF were treated with ATP $(61.3 \%)$, and 279 episodes treated with shocks $(29.37 \%)$ and overall 85 episodes $(9.4 \%)$

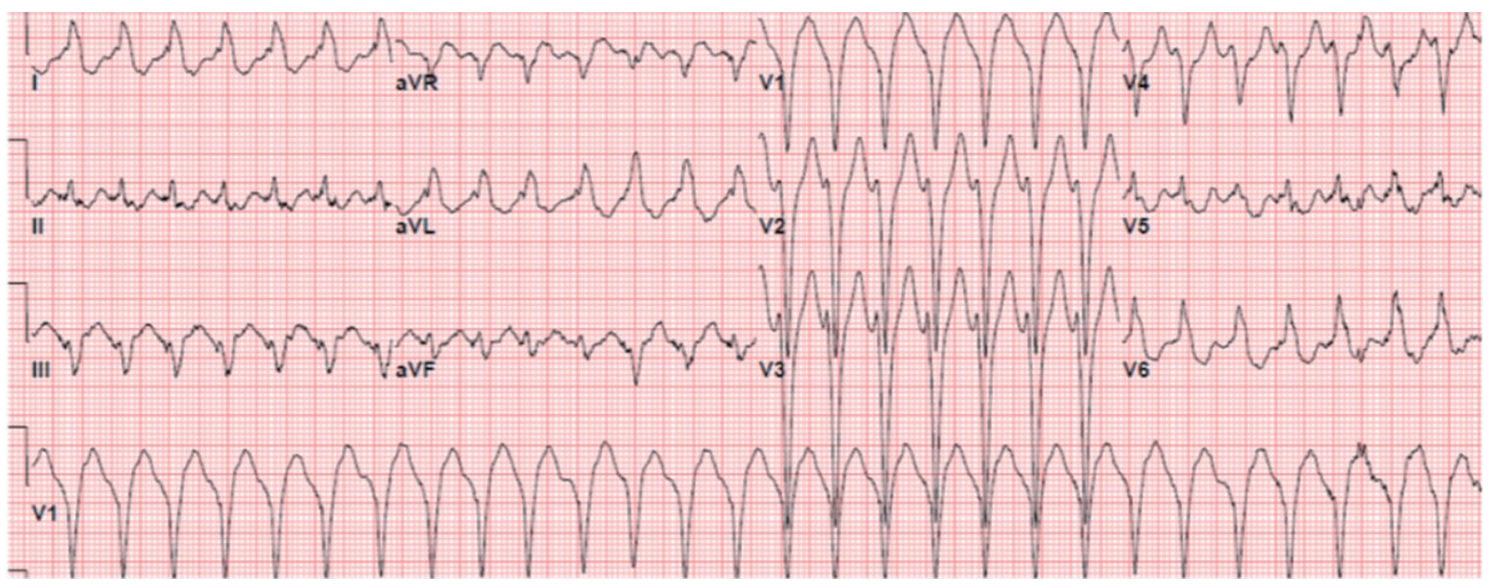

Figure 2. ECG in a patient with ARVC/D showing ventricular tachycardia with a left bundle branch block (LBBB) morphology and superior axis suggesting a RV inferior wall origin. 

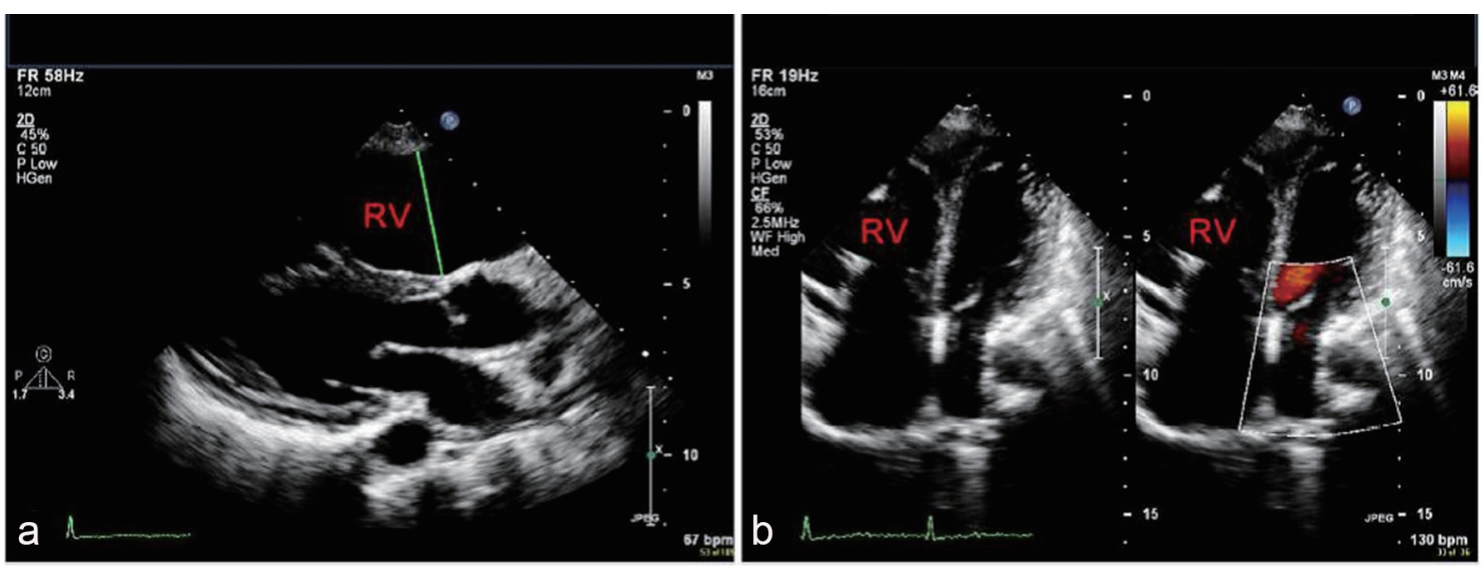

Figure 3. Parasternal long axis (a) and apical four-chamber (b) echocardiographic views showing RV dilatation [13].

were inappropriate (45 episodes treated with ATP (4.73\%), and 40 treated with shocks (4.21\%)) (Fig. 5).

In the primary prevention patients, one had appropriate $(14.3 \%)$, and one had inappropriate ICD therapy $(14.3 \%)$. In the secondary prevention patients, 10 had appropriate $(66.7 \%)$, and 4 had inappropriate ICD therapy $(26.7 \%)$. Nine patients suffered at least one electrical storm (41\%), all but one were due to VT/ VF episodes. The time to first appropriate ICD therapy ranged from 3 days to 2 years and 9 months (mean: $6 \pm 1.4$ months).

When comparing patients with appropriate ICD therapy to those with inappropriate ICD therapy, only secondary prevention was a predictor of appropriate therapy (Table 5).

The causes of inappropriate ICD therapy were atrial fibrillation (15 episodes $(17.6 \%))$, sinus tachycardia (ST) (24 episodes $(28.2 \%)$ ), supraventricular tachycardia (SVT) (36 episodes $(42.2 \%))$, lead noise (6 episodes $(7.1 \%))$ and electromagnetic interference (4 episodes (4.7\%)) (Fig. 6).

Regarding ICD-related complications, one patient had ICD lead fracture after 6.5 years, and a new ICD lead was implanted. Another patient underwent a new ICD lead implantation at the time of ICD generator change 7 years after initial implantation prophylactically as his ICD lead was a recalled lead (Medtronic Sprint Fidelis). There was no ICD system-related infection.

\section{Medical therapy}

Almost all patients were on antiarrhythmic medications. Seven patients were on sotalol (31.8\%), three on amiodarone $(13.6 \%)$, and eleven on beta-blockers $(50 \%)$. Nine patients were on angiotensin-converting enzyme inhibitors/angiotensin II receptor blockers (ACEI/ARBs) (41\%), and two on spironolactone (9\%).

\section{Electrophysiology study and ablation}

Eight patients underwent electrophysiology study (EPS); five patients underwent VT ablation, two patients had premature ventricular contraction (PVC)'s ablation and one patient had a non-inducible VT. Three patients had EPS with SVT ablation (two atrioventricular nodal re-entry tachycardia and one atrial tachycardia).

\section{Genetic testing}

Genetic testing was performed in 14 patients (five primary prevention and nine secondary prevention patients). In the primary prevention patients; three were positive (all plakophilin-2 (PKP2) gene), and two were negative. In the secondary prevention patients; seven were positive (five PKP2, one desmoplakin (DSP) and one desmocollin-2 (DSC2) genes), and two were negative.

\section{Discussion}

The ICD therapy is the only proven therapy for SCD prevention in high-risk ARVC/D patients. Nearly two-thirds of our patients experienced appropriate ICD therapy during their follow-up indicating a high prevalence of VT/VF in ARVC/D patients. The average appropriate ICD therapy was $4.8 \%$ per patient/year, and inappropriate ICD therapy or intervention occurred in about one-fourth of the patients with an average of $1.5 \%$ per patient/year. In a recent meta-analysis, it is found that the annualized appropriate ICD intervention rate in ARVC/D patients was $9.5 \%$, and the annualized inappropriate ICD intervention rate was $3.7 \%$ [14].

Fortunately, ATP therapy was very effective in terminating most of the VT episodes in our patients. This is compatible with what has been found in previous studies where ATP attempt has been shown to be effective in terminating about $84.4 \%$ of VT episodes [15].

Patients with secondary prevention indication had more appropriate ICD therapy compared to those with primary prevention indication $(66.7 \%$ versus $14 \%)$, and there was no significant difference in appropriate and inappropriate ICD therapies in those with positive or negative genetic testing. In a large cohort of ARVC/D patients and family members, appropriate ICD therapy in secondary prevention patients was $84 \%$ and $71 \%$ in 
Table 3. Summary of Echocardiogram Changes

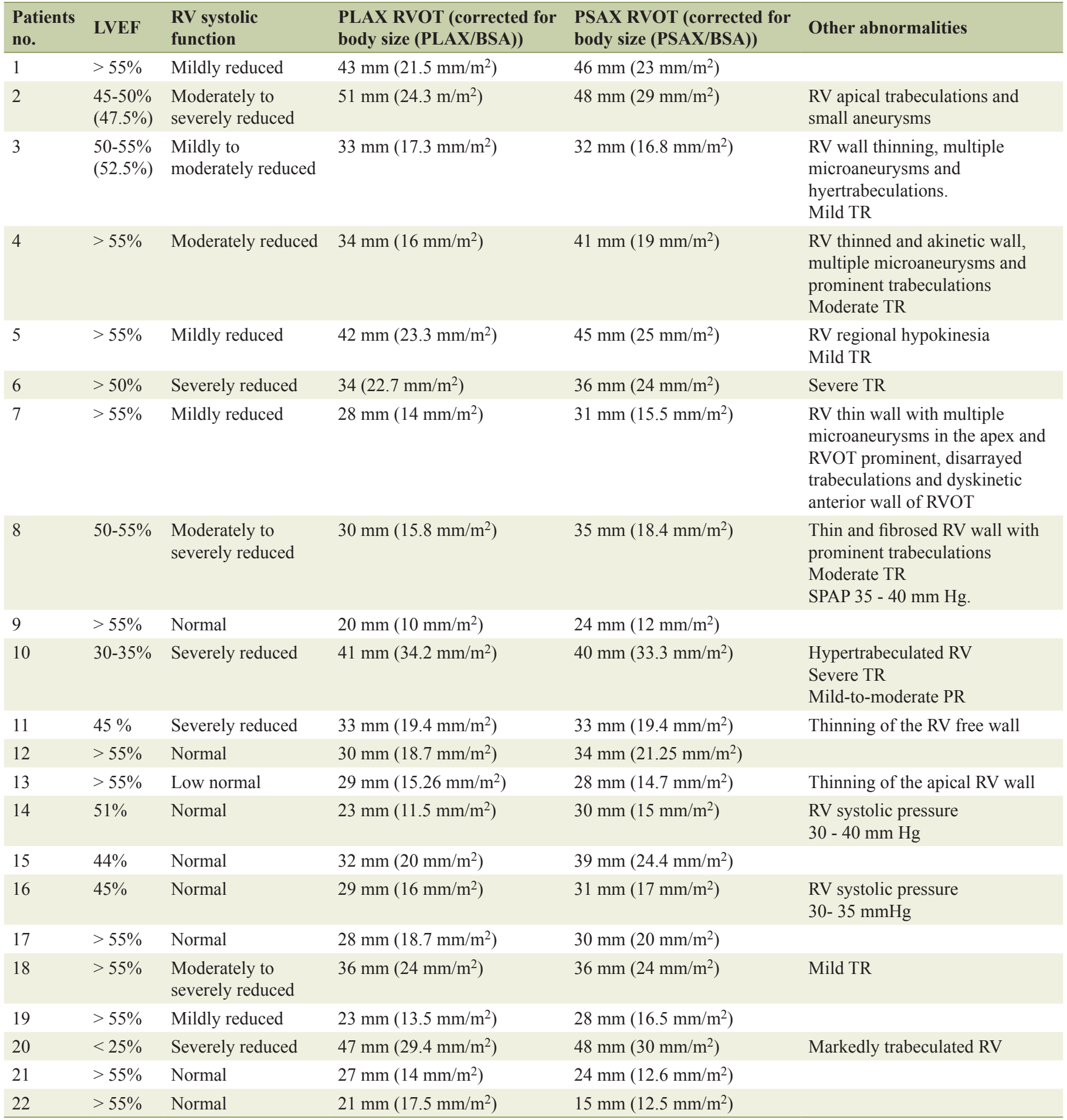

BSA: body surface area; LVEF: Left ventricular ejection fraction; mm: millimeters; $m^{2}$ : square meter; PLAX: parasternal long-axis view; PSAX: parasternal short-axis view; PR: pulmonic valvular regurgitation; RV: right ventricle; RVOT: RV outflow tract; SPAP: systolic pulmonary artery pressure; TR: tricuspid regurgitation. For the 2D echo major and minor criteria values, see reference [4].

those with and without genetic mutation respectively [7]. On the other hand, appropriate ICD therapy in primary prevention patients was $16 \%$ and $29 \%$ in the two groups, respectively [7].
The most common cause of inappropriate ICD therapy in this series is SVT, followed by ST and both accounts for about $70 \%$ of inappropriate ICD therapies. This is likely due 

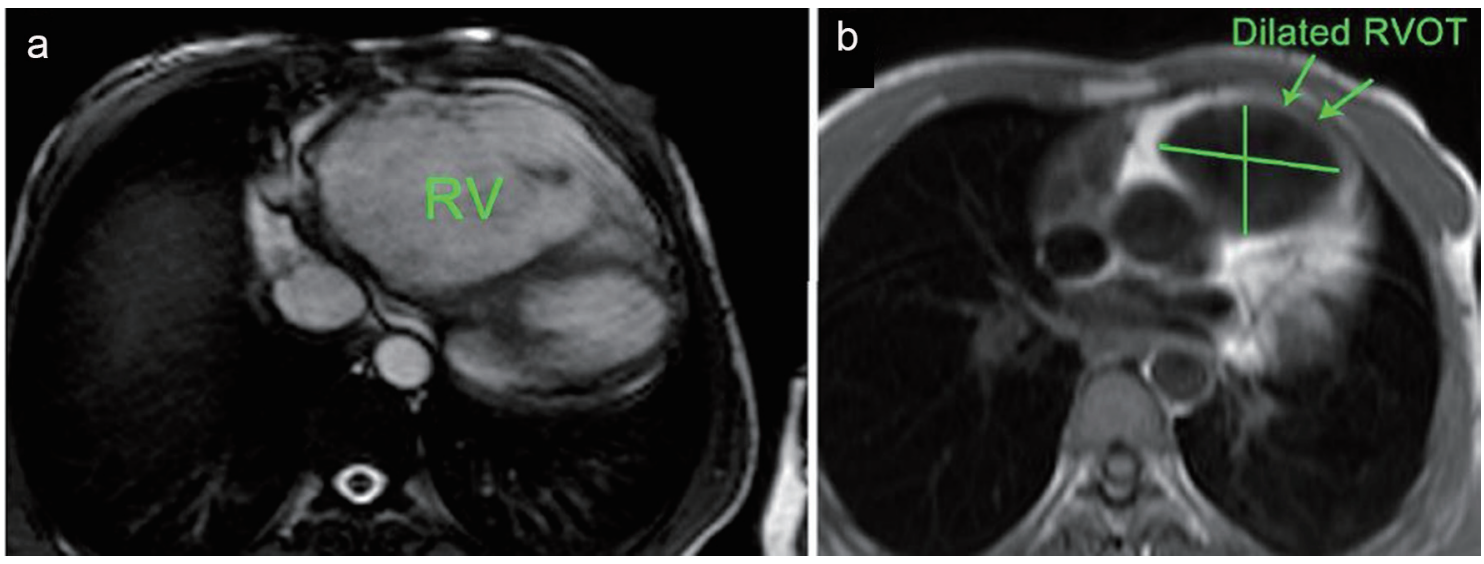

Figure 4. (a) Axial cine SSFP (steady-state free precession) MRI showing significant RV dilatation and (b) black-blood-prepared HASTE (half-Fourier acquired single-shot turbo spin echo) axial slice MRI at level of RVOT showing RVOT dilatation [13].

Table 4. Summary of Cardiac Magnetic Resonance Imaging (MRI) Changes

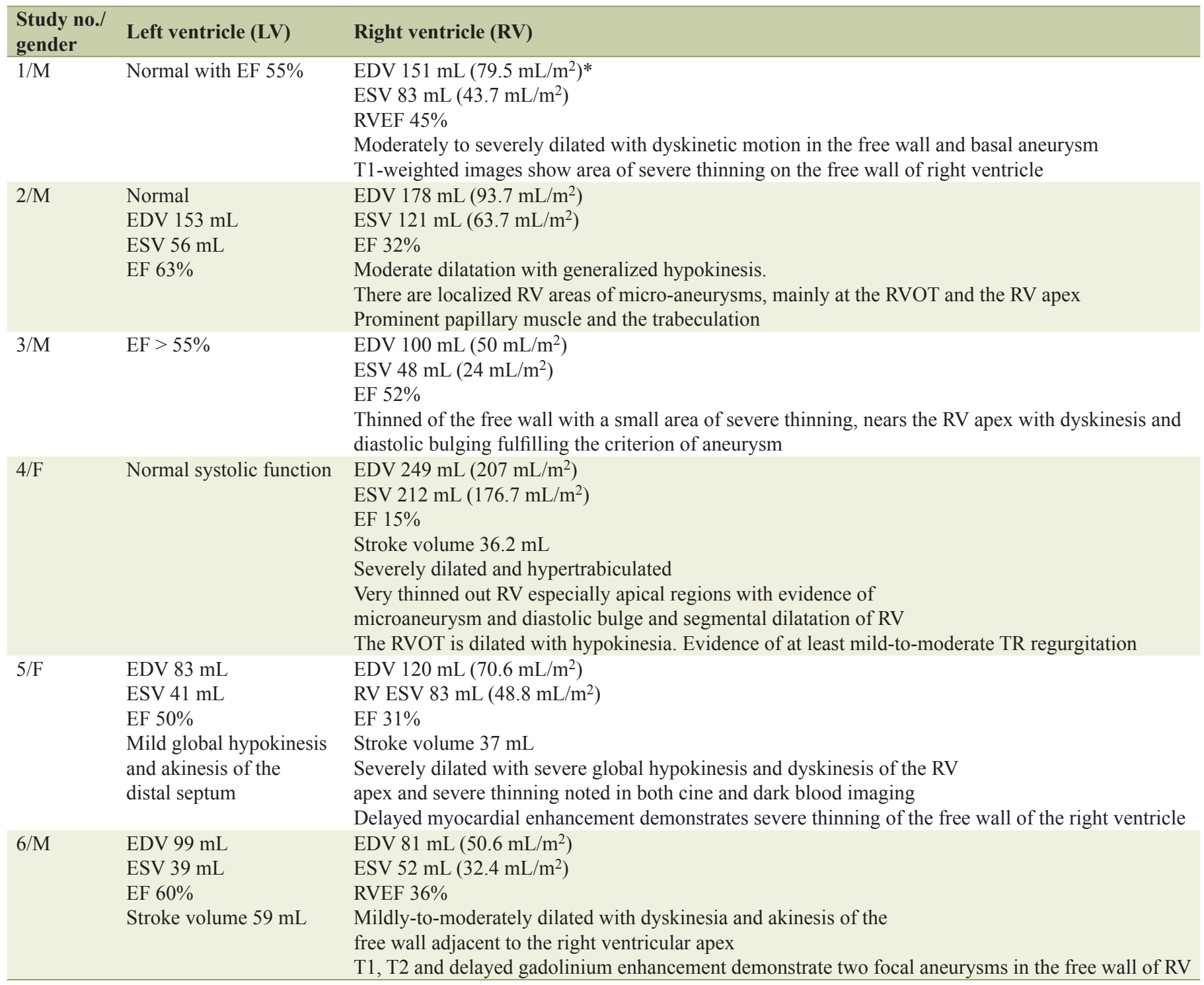


Table 4. Summary of Cardiac Magnetic Resonance Imaging (MRI) Changes - (continued)

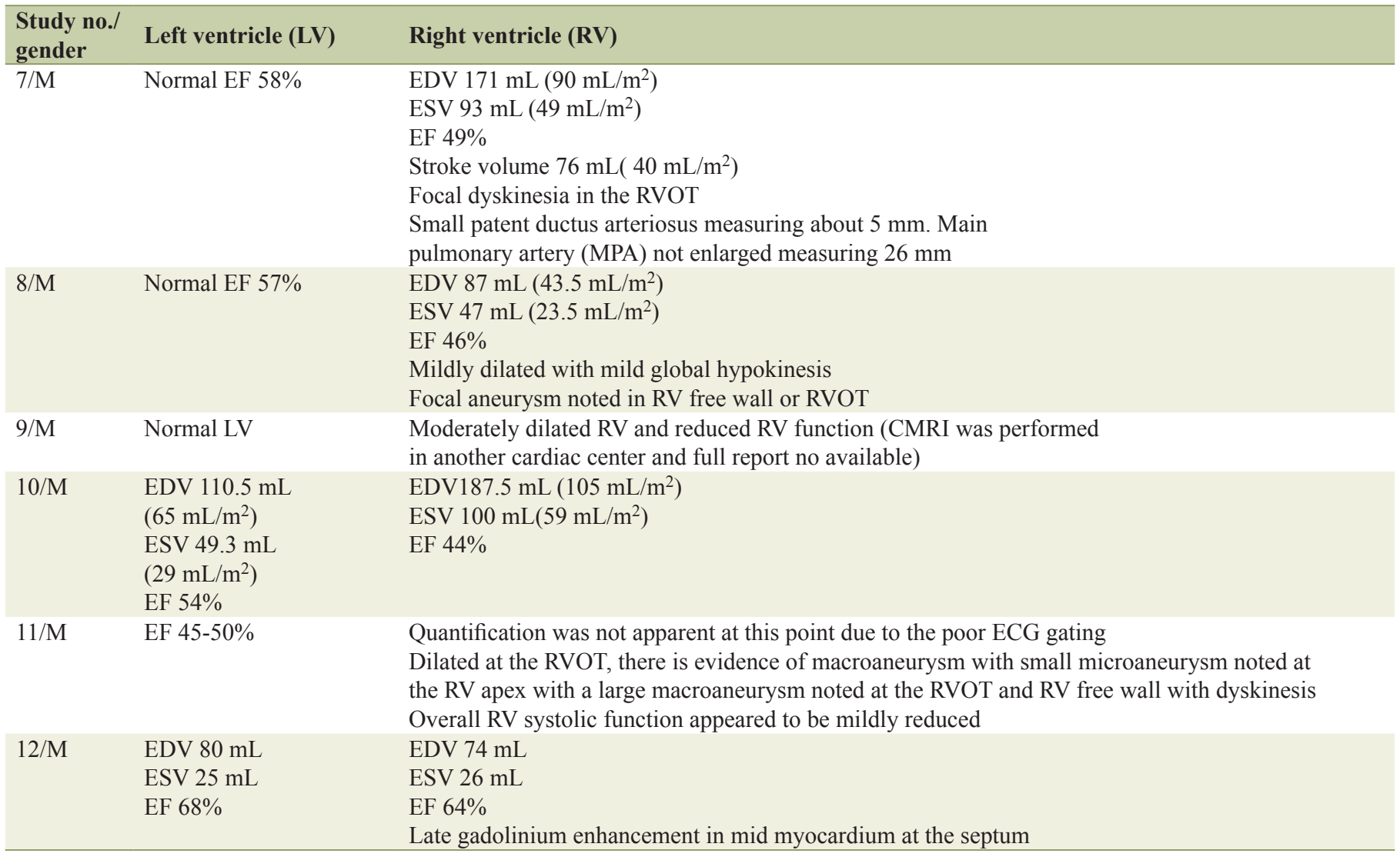

*Between practices values corrected for body size (value/body surface area (BSA)). EF: ejection fraction; EDV: end-diastolic volume; ESV: endsystolic volume mm millimeters; $\mathrm{m}^{2}$ : square meter, TR: tricuspid valve. For the cardiac MRI major and minor criteria values, see reference [4].

to the young age at implantation. Atrial fibrillation accounts for $17.6 \%$ of these therapies. This is compatible with what has been reported before [14].

In this small cohort, we did not find significant difference in the incidence of inappropriate ICD therapy between single and dual chamber ICD. Currently, there is no clear evidence to determine whether a single chamber or a dual chamber ICD is preferable for patients with ARVD/C [16].

Although subcutaneous ICD (S-ICD) has been used in patients with ARVC/D, the lack of ATP therapy at current S-ICD devices makes it less attractive to this group of patients. Furthermore, the negative T-waves (NTWs) in the right precordial leads may partially or completely revert with exercise in most patients with ARVC/D [17], and this may result in the lack of consistency of an appropriate sensing vector both at resting and during exercise [18], or inappropriate ICD detection and therapy [19]. However, a software update to improve the sensing of the device may minimize this problem [19].

\section{Study limitations}

The main limitations of this study are the retrospective nature and the small sample size. However, the ARVC/D is a rare disease and the mean follow-up in this study is longer than previ- ous studies with a mean follow-up of about nine and half years.

\section{Conclusions}

ARVC/D patients are at high risk of VT/VF, and ICD therapy is an effective management strategy in terminating these arrhythmias. Most of ICD therapies in ARVC/D patients are appropriate. Appropriate ICD programming and use of ATP will reduce the risk of having appropriate and inappropriate ICD shocks. Although we do not have any patient with subcutaneous ICD in this study, the high success rate of ATP suggests that transvenous ICD would be more appropriate in ARVC/D patients.

\section{Conflict of Interest}

The authors declare no conflict of interest related to this article.

\section{Financial Support}

No source of funding to declare. 


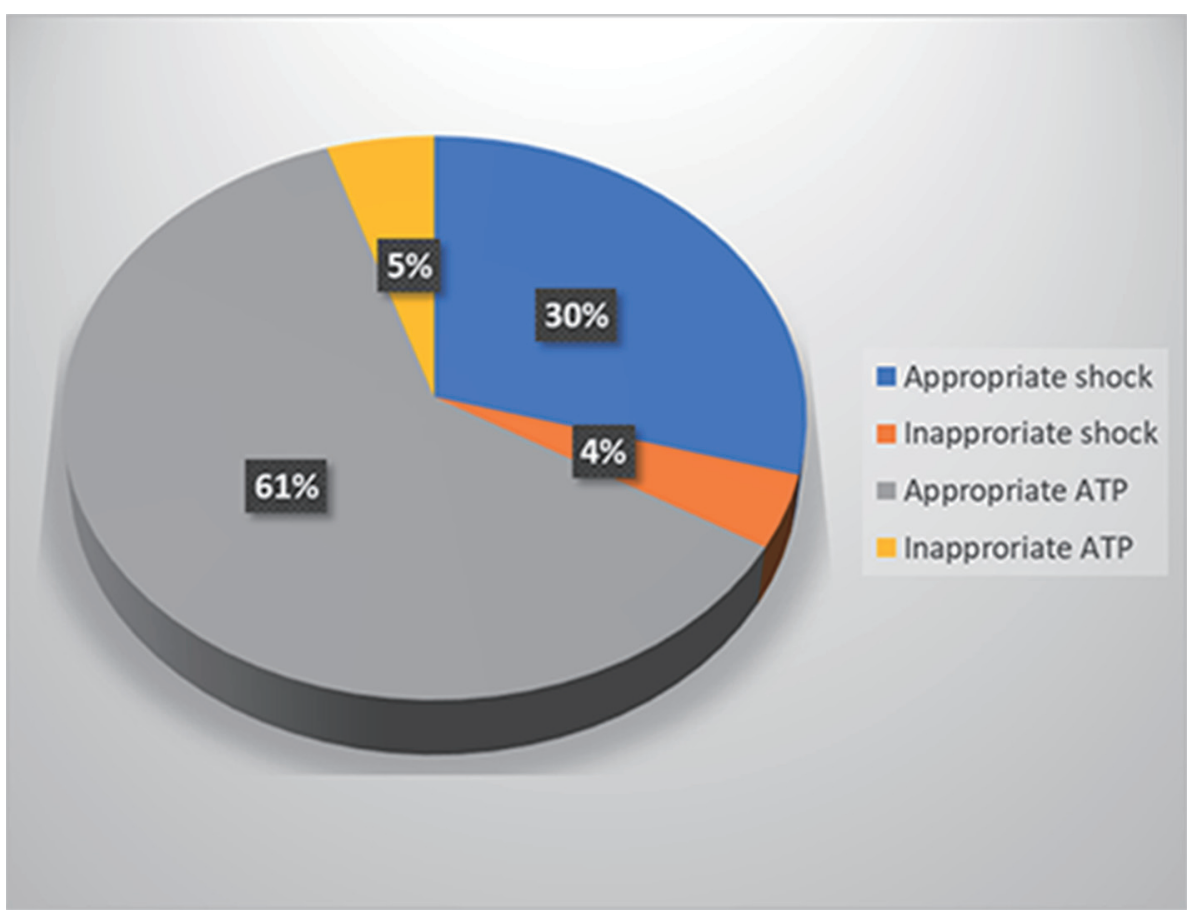

Figure 5. Implantable cardioverter defibrillator (ICD) therapy classification. ATP: antitachycardia pacing.

Table 5. Comparison Between Patients With Appropriate and Inappropriate Implantable Cardioverter Defibrillator (ICD) Therapy

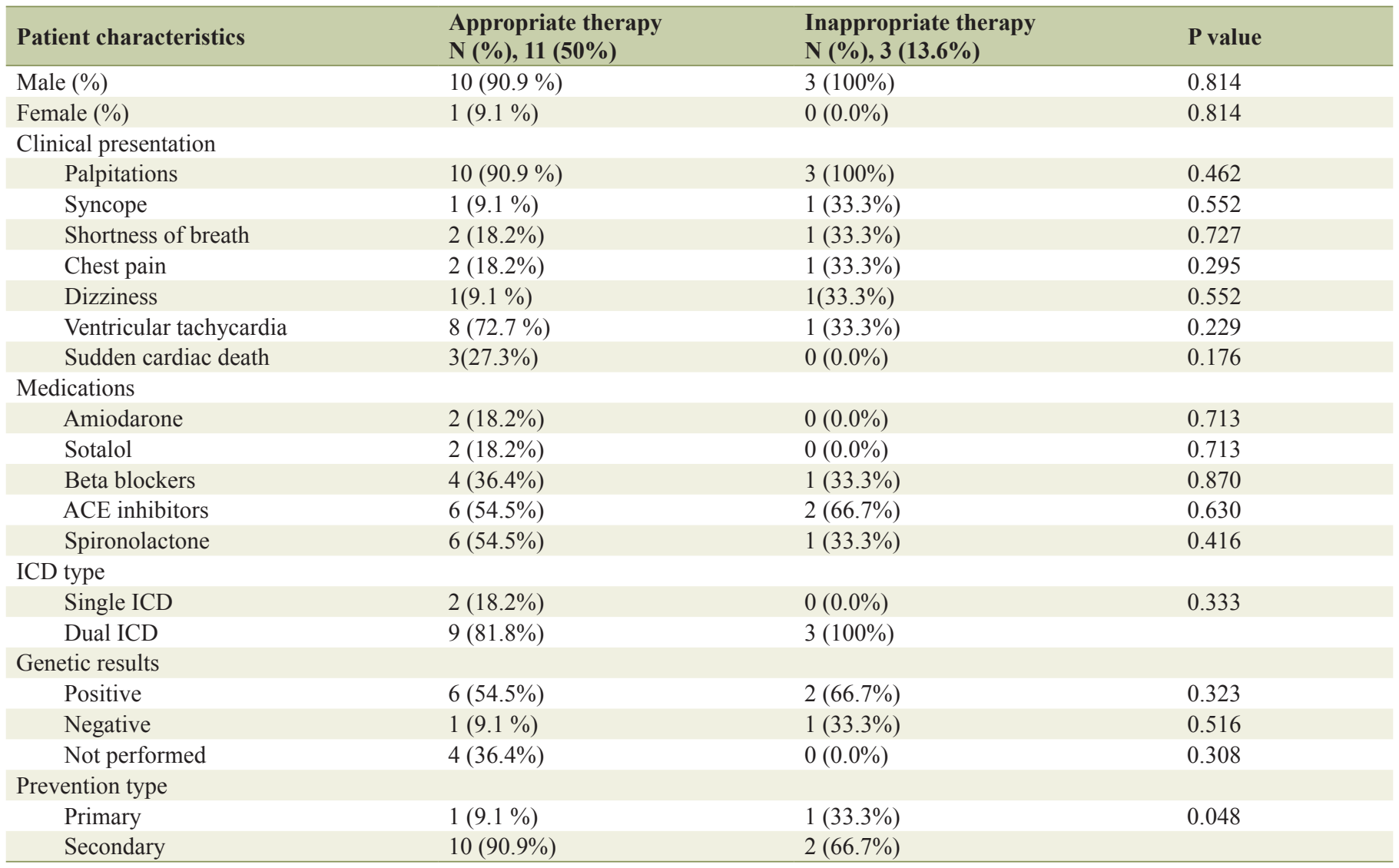

ACE: angiotensin-converting enzyme; ICD: implantable cardioverter defibrillator. 


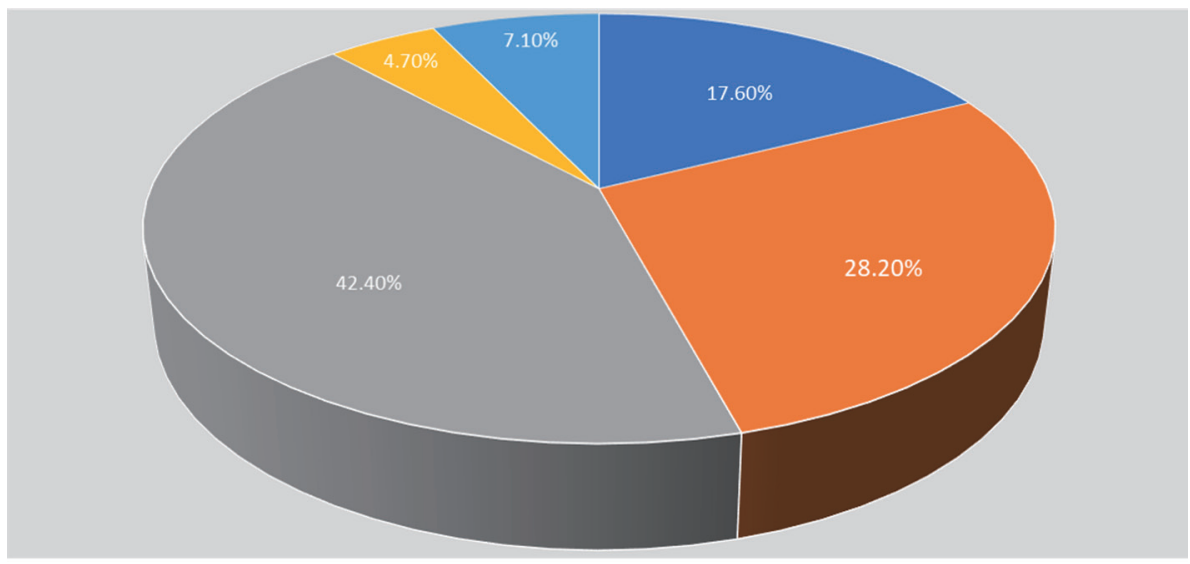

- $\mathrm{AF}=\mathrm{ST}=\mathrm{SVT}=\mathrm{EMI} \|$ Lead noise

Figure 6. Causes of inappropriate implantable cardioverter defibrillator (ICD) therapy. AF: atrial fibrillation; EMI: elecromanganic interference; ST: sinus tachycardia; SVT: supraventricular tachycardia.

\section{References}

1. Marcus FI, Fontaine GH, Guiraudon G, Frank R, Laurenceau JL, Malergue C, Grosgogeat Y. Right ventricular dysplasia: a report of 24 adult cases. Circulation. 1982;65(2):384-398.

2. Corrado D, Basso C, Thiene G, McKenna WJ, Davies MJ, Fontaliran F, Nava A, et al. Spectrum of clinicopathologic manifestations of arrhythmogenic right ventricular cardiomyopathy/dysplasia: a multicenter study. J Am Coll Cardiol. 1997;30(6):1512-1520.

3. McKenna WJ, Thiene G, Nava A, Fontaliran F, Blomstrom-Lundqvist C, Fontaine G, Camerini F. Diagnosis of arrhythmogenic right ventricular dysplasia/cardiomyopathy. Task Force of the Working Group Myocardial and Pericardial Disease of the European Society of Cardiology and of the Scientific Council on Cardiomyopathies of the International Society and Federation of Cardiology. Br Heart J. 1994;71(3):215-218.

4. Marcus FI, McKenna WJ, Sherrill D, Basso C, Bauce B, Bluemke DA, Calkins H, et al. Diagnosis of arrhythmogenic right ventricular cardiomyopathy/dysplasia: proposed modification of the Task Force Criteria. Eur Heart J. 2010;31(7):806-814.

5. Thiene G, Nava A, Corrado D, Rossi L, Pennelli N. Right ventricular cardiomyopathy and sudden death in young people. N Engl J Med. 1988;318(3):129-133.

6. Corrado D, Leoni L, Link MS, Della Bella P, Gaita F, Curnis A, Salerno JU, et al. Implantable cardioverter-defibrillator therapy for prevention of sudden death in patients with arrhythmogenic right ventricular cardiomyopathy/dysplasia. Circulation. 2003;108(25):3084-3091.

7. Groeneweg JA, Bhonsale A, James CA, te Riele AS, Dooijes D, Tichnell C, Murray B, et al. Clinical presentation, long-term follow-up, and outcomes of 1001 arrhythmogenic right ventricular dysplasia/cardiomyopathy patients and family members. Circ Cardiovasc Genet.
2015;8(3):437-446.

8. Maron BJ, Shen WK, Link MS, Epstein AE, Almquist AK, Daubert JP, Bardy GH, et al. Efficacy of implantable cardioverter-defibrillators for the prevention of sudden death in patients with hypertrophic cardiomyopathy. N Engl J Med. 2000;342(6):365-373.

9. Credner SC, Klingenheben T, Mauss O, Sticherling C, Hohnloser SH. Electrical storm in patients with transvenous implantable cardioverter-defibrillators: incidence, management and prognostic implications. J Am Coll Cardiol. 1998;32(7):1909-1915.

10. Myerburg RJ, Castellanos A. Cardiac Arrest and Sudden Cardiac Death. In: Bonow RO, Mann DL, Zipes DP, Libby P, Braunwald E, Eds., Braunwald's Heart Disease: A Textbook of Cardiovascular Medicine, Elsevier Saunders, Philadelphia, 2012. 845-884.

11. Chugh SS, Teodorescu C, Evanado A, Reiner K. Sudden Unexplained Death in the Community. In: Brugada R, Brugada J, Brugada P, Eds, Clinical Approach to Sudden Cardiac Death Syndromes, Springer Verlag, London, 2010. 1-6.

12. Russo AM, Stainback RF, Bailey SR, Epstein AE, Heidenreich PA, Jessup M, Kapa S, et al. ACCF/HRS/AHA/ ASE/HFSA/SCAI/SCCT/SCMR 2013 appropriate use criteria for implantable cardioverter-defibrillators and cardiac resynchronization therapy: a report of the American College of Cardiology Foundation appropriate use criteria task force, Heart Rhythm Society, American Heart Association, American Society of Echocardiography, Heart Failure Society of America, Society for Cardiovascular Angiography and Interventions, Society of Cardiovascular Computed Tomography, and Society for Cardiovascular Magnetic Resonance. J Am Coll Cardiol. 2013;61(12):1318-1368.

13. Bandar Al-Ghamdi (April 12th 2017). Arrhythmogenic Right Ventricular Cardiomyopathy/Dysplasia, Cardiomyopathies Kaan Kirali, IntechOpen. Available from: 
https://www.intechopen.com/books/cardiomyopathiestypes-and-treatments/arrhythmogenic-right-ventricularcardiomyopathy-dysplasia.

14. Schinkel AF. Implantable cardioverter defibrillators in arrhythmogenic right ventricular dysplasia/cardiomyopathy: patient outcomes, incidence of appropriate and inappropriate interventions, and complications. Circ Arrhythm Electrophysiol. 2013;6(3):562-568.

15. Gulizia MM, Piraino L, Scherillo M, Puntrello C, Vasco C, Scianaro MC, Mascia F, et al. A randomized study to compare ramp versus burst antitachycardia pacing therapies to treat fast ventricular tachyarrhythmias in patients with implantable cardioverter defibrillators: the PITAGORA ICD trial. Circ Arrhythm Electrophysiol. 2009;2(2):146-153.

16. Roguin A, Bomma CS, Nasir K, Tandri H, Tichnell C, James C, Rutberg J, et al. Implantable cardioverter-de- fibrillators in patients with arrhythmogenic right ventricular dysplasia/cardiomyopathy. J Am Coll Cardiol. 2004;43(10):1843-1852.

17. Zorzi A, ElMaghawry M, Rigato I, Cardoso Bianchini F, Crespi Ponta G, Michieli P, Migliore F, et al. Exercise-induced normalization of right precordial negative $T$ waves in arrhythmogenic right ventricular cardiomyopathy. Am J Cardiol. 2013;112(3):411-415.

18. Migliore F, Bertaglia E, Zorzi A, Corrado D. Subcutaneous Implantable Cardioverter-Defibrillator and Arrhythmogenic Right Ventricular Cardiomyopathy: The Importance of Repeat ECG Screening During Exercise Test. JACC Clin Electrophysiol. 2017;3(7):785-786.

19. Allocca G, Sitta N, Turiano G. Inappropriate shocks by subcutaneous defibrillator in a patient with arrhythmogenic right ventricular cardiomyopathy: problem fixed. Europace. 2015;17(7):1067. 